\title{
Emerging Magnetism in Platinum Nanowires
}

\author{
A. Delin ${ }^{1}$ and E. Tosatti ${ }^{1,2,3}$ \\ ${ }^{1}$ Abdus Salam International Center for Theoretical Physics (ICTP), Strada Costiera \\ 11, 34100 Trieste, Italy \\ ${ }^{2}$ International School for Advanced Studies (SISSA), via Beirut 2-4, 34014 Trieste, \\ Italy \\ ${ }^{3}$ INFM DEMOCRITOS National Simulation Center, via Beirut 2-4, 34014 Tri- \\ este, Italy
}

\begin{abstract}
We have investigated infinitely long, monostrand Pt nanowires theoretically, and found that they exhibit Hund's rule magnetism. We find a spin moment of $0.6 \mu_{B}$ per atom, at the equilibrium bond length. Its magnetic moment increases with stretching. The origin of the wire magnetism is analyzed and its effect on the conductance through the wire is discussed.
\end{abstract}

Keywords: Density functional calculations, Magnetic phenomena, Platinum, Nanowires 


\section{Introduction}

Little is currently understood about how magnetism arises at the nanoscale and how it affects the properties of nanosized metallic objects. Systems of special interest in this context are nanowires and atomic-sized nanocontacts. The one-dimensional (1D) character of such systems causes specific physical phenomena to appear, most notably quantized ballistic conductance. [1]

These phenomena will be heavily affected by the possible presence of magnetism in the nanosystem, especially of a genuine Hund's rule magnetic order parameter. Here, we report theoretical studies of magnetism in $\mathrm{Pt}$ monowires, i.e., wires consisting of a single line of equally spaced Pt atoms. Such objects, several atoms long, have recently been observed by Rodrigues et $a l .[2]$

Pt is a transition metal, with a partially filled $5 d$ shell. The free Pt atom has a $d^{9} s^{1}$ configuration, giving a total magnetic moment of $2 \mu_{B}$, and therefore it is reasonable to expect that a sufficiently stretched wire should eventually show some type of magnetic ordering. In particular, at the localized side of a Mott transition, one could expect an antiferromagnetic ordering. In the bulk metal, the Pt $5 d$ band is too wide to provoke spin-polarization and there is no evidence of Pt showing surface magnetism either. 3 . Yet, the $5 d$ band is still only partially occupied, and the density of states at the Fermi level is quite high. It appears therefore to be an open question whether Pt nanowires might exhibit ferromagnetic ordering. If that were the case, it might result in interesting phenomena such as spin-polarized current flow and magneticfield dependent conductance, phenomena that could prove highly useful in spintronics applications. 
Of course, thermal fluctuations, very large in a nanosystem, will generally act to destroy static magnetic order in the absence of an external field. Sufficiently slow fluctuations transform a nanomagnet to a superparamagnetic state, where magnetization fluctuates on some time scale, between equivalent magnetic valleys, separated, e.g., by anisotropy-induced energy barriers. If the barriers are sufficiently large - and the fluctuations sufficiently slow the nanosystem spends most of the time in a single magnetic valley, and will for many practical purposes behave as magnetic. We may in these circumstances be allowed to neglect fluctuations altogether, and to approximate the calculated properties of the superparamagnetic nanosystem with those of a statically magnetized one. Experimentally, evidence of 1D superparamagnetism with fluctuations sufficiently slow on the time scale of the probe has been reported in Co atomic chains deposited at Pt surface steps. [4]

\section{Method}

The density-functional calculations [5] reported here are all-electron, in order to rule out possible sources of doubt that might arise when using pseudopotentials in presence of magnetism and in a nonstandard configurations. [6] We employed an all-electron full-potential linear muffin-tin orbital (FP-LMTO) basis set [7] together with a generalized gradient approximation (GGA) [8] to the exchange-correlation functional. As a double check, some of the calculations were repeated using the linear augmented plane-wave (LAPW) code WIEN97. [9] None of these calculations assume any shape approximation of the potential or wave functions. 
We performed both scalar relativistic (SR) calculations, and calculations including the spin-orbit coupling as well as the scalar-relativistic terms. The latter will be referred to as "fully relativistic" (FR) calculations in the following, although we are not strictly solving the full Dirac equation, or making use of current density functional theory. In the FR calculations, the spin axis was chosen to be aligned along the wire direction.

The calculations were performed with inherently three-dimensional codes. Thus, the infinitely long, straight, isolated monatomic nanowire was simulated by a regular array of well-spaced nanowires. Convergence of the magnetic moment was checked with respect to k-point mesh density, Fourier mesh density, tail energies, and wire-wire vacuum distance.

\section{Results and Discussion}

The magnetic spin moment per atom monowire as a function of bond length is shown in Fig. 1. The solid line refers to the fully relativistic calculation (FR), and the dotted line to the scalar relativistic (SR) calculation. As seen, the magnetic profiles for the SR and FR calculations are very different. The SR calculation for Pt predicts this metal to be magnetic only for wire bond lengths larger than around $2.7 \AA$, which corresponds to a rather stretched wire, whereas the FR calculation predicts it to be magnetic in the whole range of bond lengths plotted $\left(2.2 \AA\right.$ to $3.2 \AA$ ), with a moment of $0.6 \mu_{B}$

per atom at the equilibrium bond length $2.48 \AA$. For sufficiently large bond lengths (in the interval shown in the figure) the magnetic moment reaches a plateau value, still well below the atomic spin moment $2 \mu_{B}$. For even larger 
bond lengths (not shown), the magnetic moment eventually approaches the atomic spin moment $2 \mu_{B}$. The energy gain per atom due to spin polarization is rather small, around $8 \mathrm{meV}$ for the FR calculation at the equilibrium bond length $2.48 \AA$. Antiferromagnetic Pt monowire configurations were also tested for bond lengths around the equilibrium one, and found to be energetically unstable compared to the ferromagnetic configuration.

Several mechanisms, some favoring and other disfavoring a spin-polarized ground state, are at work in the wire. The number of nearest neighbors is only two in the wire, compared to 12 in bulk. This reduction of the number of nearest neighbors in the wire compared to the bulk causes a narrowing of the $5 d$ band, and the band width may become sufficiently small that the gain in exchange energy due to spin polarization is larger than the increase in kinetic energy. On the other hand, the equilibrium bond length is significantly smaller in the wire, $10 \%$ smaller than in bulk, which partly counteracts the band-narrowing effect of the reduced number of neighbors.

Highly important in this context are the very sharp van Hove singularities caused by the one-dimensionality of the system. These give rise to a very high density of states at the Fermi level when a van Hove singularity is sufficiently close to the Fermi level, which in turn results in a Stoner product larger than one, and thus spontaneous spin-polarization.

In order to provide a more detailed analysis of the origin of the magnetism in the wires, we found it useful to analyze the wire band structures. Fig. 2 shows FR band structures of the Pt monowire for several different bond lengths. The bands run from the zone center, $\Gamma$, to the zone edge, $A$, in the direction of the wire. Fig. 3 shows the corresponding SR band structures. 
The sharp van Hove singularities manifest themselves in the band structures through the horizontal band edges at the zone edge $\mathrm{A}$ and zone center $\Gamma$. Since the orbital character is of critical importance for the moment formation, it is useful to divide the band structure into distinct orbitals - illustrated in Fig. (4f for the FR case. This figure has four panels, displaying separately the $s$, $d_{z},\left(d_{x z}, d_{y z}\right)$ and $\left(d_{x y}, d_{x^{2}-y^{2}}\right)$ characters of the bands. The vertical error bars, or "thickness", of the bands indicate the relative character weight. In the SR case (not shown), the $\left(d_{x z}, d_{y z}\right)$ and $\left(d_{x y}, d_{x^{2}-y^{2}}\right)$ orbitals correspond each to separate bands, and the $s$ and $d_{z}$ orbitals hybridize, forming two bands of high dispersion. With the help of Fig. 固, we can immediately recognize an important mechanism that favors spin-polarization in the wire. As seen, the bands have mostly $d$ character at the edges, and therefore the exchange energy gain will be rather large if a band spin-splits so that one of the spinchannel band edges ends up above the Fermi level, and the other one below. ${ }^{1}$ Thus, if a band edge ends up sufficiently near the Fermi level, we may expect a magnetic moment to develop. While apparently similar to the magnetization of the jellium wire, [11 magnetism here is much more substantial, since the $d$ states involve a much stronger Hund's rule exchange. We say that the magnetism exhibited in the Pt wire is Hund's rule magnetism, in order to differentiate it from the situation in the jellium wire.

By comparing the magnetic and nonmagnetic band structures, we see that the relatively flat $\left(d_{x y}, d_{x^{2}-y^{2}}\right)$ bands play the leading role in the formation of

\footnotetext{
${ }^{1}$ Strictly speaking, the spin-orbit coupling will mix the two spin channels so that, in general, an eigenvalue will have both majority and minority spin character. However, we found that this mixing is so small, typically just a few percent, that it is irrelevant for the qualitative discussion we make here.
} 
the magnetic state. In the SR band structures, these bands (all degenerate) sit well below the Fermi level, between $-1 \mathrm{eV}$ and $-0.2 \mathrm{eV}$ for the equilibrium bond length, and thus cannot contribute to spin polarization. For larger bond lengths than around $2.6 \AA$, the edge at A moves critically close to the Fermi level, and the bands split. In the FR calculations, however, a band edge at A of this symmetry is pinned to the Fermi level in the whole range of studied bond lengths, down to $2.2 \AA$, explaining the large difference in magnetic profile between the SR and FR calculations. This band edge is close to the Fermi level in the FR calculation but not in the SR calculation simply because in the FR calculation, the $\left(d_{x y}, d_{x^{2}-y^{2}}\right)$ orbital hybridizes partly with the $\left(d_{x z}, d_{y z}\right)$ orbital, with the result that the $\left(d_{x y}, d_{x^{2}-y^{2}}\right)$ band splits into an upper part of purely $\left(d_{x y}, d_{x^{2}-y^{2}}\right)$ character, and a lower part hybridizing strongly with the $\left(d_{x y}, d_{x^{2}-y^{2}}\right)$ orbital, thereby splitting up into two bands. If the average position of all the $\left(d_{x y}, d_{x^{2}-y^{2}}\right)$ levels is taken, we end up more or less where the SR $\left(d_{x y}, d_{x^{2}-y^{2}}\right)$ orbital originally was, energy-wise. Thus, one net effect of the spin-orbit coupling is that it increases the energy of part of the $\left(d_{x y}, d_{x^{2}-y^{2}}\right)$ orbital. This also explains why the magnetic moment of the FR calculation is smaller than in the SR calculation in the bond-length range $2.7 \AA$ to $3.1 \AA$.

Three other band edges, a $d_{z^{2}}$-dominated one located at $\mathrm{A}$ and the $\left(d_{x z}, d_{y z}\right)$ dominated ones with located at $\Gamma$ are also important, and add to the size of the magnetic moment as they split around the Fermi level once the spinpolarization has been triggered.

The magnetic or superparamagnetic state of a small piece of nanowire bridging between nonmagnetic tips might at first seem very problematic to 
detect. It should in fact be detectable by measuring ballistic conductance as a function of both temperature and of an external magnetic field. The field, even a modest one depending on temperature, can drive a superparamagnetic wire from thermally disordered to fully spin polarized. In this polarized state, the nature and number of current-carrying channels, each corresponding to a band crossing the Fermi level, will differ from that of the nonmagnetic state. The channel number characteristic of the magnetic state should in fact last well into the superparamagnetic state at zero field. In the case of our infinitely long Pt monowire, we find that the nonmagnetic wire has 10 open conductance channels and the spin-polarized 8, which would correspond to a Landauer conductance of $5 G_{0}$ and $4 G_{0}$, respectively, if all channels conducted fully. ( $G_{0}=2 e^{2} / h$ is the fundamental conductance quantum.) However, the bands splitting around the Fermi level due to the magnetic state are of mainly $d$-character, which conduct poorly compared to $s$ channels. This means that the conductance of the magnetic and nonmagnetic wires will be much lower than these numbers indicate, and the difference in conductance will be significantly smaller than $G_{0}$. Conductance calculations for a magnetic Pt nanowire segment between tips are presently being considered, but are beyond the scope of this work.

In summary, we find that infinitely long $\mathrm{Pt}$ monowires have a ferromagnetic ground state, with a moment of around $0.6 \mu_{B}$ at the equilibrium bond length $2.48 \AA$. The moment increases with stretching, and the trigger of the moment formation is the $\left(d_{x y}, d_{x^{2}-y^{2}}\right)$ orbital. The resulting superparamagnetic state of the nanowire will show up in the ballistic conductance in the form of a strong and unusual magnetic field and temperature dependence. 
Also, more majority bands cross the Fermi level than do minority bands, resulting in a partial spin-polarization of the transmitted electron current. If this current could be measured, it would be a very direct way of confirming the existence of a superparamagnetic state. Rodrigues et al. 2] recently measured the charge conductance of Pt nanocontacts and found features above as well as below $G_{0}$. More theory work will be needed to address their data, explicitly including such elements as tips and temperature.

\section{Acknowledgments}

A.D. acknowledges financial support from the European Commission through contract no. HPMF-CT-2000-00827 Marie Curie fellowship, STINT (The Swedish Foundation for International Cooperation in Research and Higher Education), and NFR (Naturvetenskapliga forskingsrådet). Work at SISSA was also sponsored through TMR FULPROP, MUIR (COFIN and FIRB RBAU01LX5H) and by INFM/F. Ruben Weht is acknowledged for discussions, and for double-checking some of the calculations using the WIEN97 code. J. M. Wills is acknowledged for letting us use his FP-LMTO code. We are also grateful to D. Ugarte for sharing with us the unpublished results of Ref. [2].

\section{References}

[1] B. J. van Wees, H. van Houten, C. W. J. Beenakker, J. G. Williamson, L. P. Kouwenhoven, D. van der Marel, and C. T. Foxon, Phys. Rev. Lett. 60, 848 (1988). 
[2] V. Rodrigues, J. Bettini, P. C. Silva, and D. Ugarte, preprint.

[3] S. Blügel, Phys. Rev. Lett. 68, 851 (1992).

[4] P. Gambardella, A. Dallmeyer, K. Maiti, M. C. Malagoli, W. Eberhardt, K. Kern, and C. Carbone, Nature 416, 301 (2002).

[5] P. Hohenberg and W. Kohn, Phys. Rev. 136, B864 (1964); W. Kohn and L. J. Sham, Phys. Rev. 140, A1133 (1965).

[6] S. R. Bahn and K. W. Jacobsen, Phys. Rev. Lett. 87, 266101 (2001).

[7] J. M. Wills, O. Eriksson, M. Alouani, and O. L. Price, in Electronic Structure and Physical Properties of Solids, edited by H. Dreyssé (Springer, Berlin, 2000).

[8] J. P. Perdew, in Electronic Structure of Solids 1991, edited by P. Ziesche and H. Eschrig (Akademie Verlag, Berlin, 1991). J. P. Perdew, K. Burke, and M. Ernzerhof, Phys. Rev. Lett. 77, 3865 (1996); J. P. Perdew, K. Burke, and M. Ernzerhof, Phys. Rev. Lett. 78, 1396 (1997); Y. Zhang and W. Yang, Phys. Rev. Lett. 80, 890 (1998); J. P. Perdew, K. Burke, and M. Ernzerhof, Phys. Rev. Lett. 80, 891 (1998).

[9] P. Blaha, K. Schwarz, and J. Luitz, computer code WIEN97 (Vienna University of Technology, Vienna, 1997). [Improved and updated UNIX version of the original copyrighted WIEN code, which was published by P. Blaha, K. Schwarz, P. Sorantin, and S. B. Trickey, Comput. Phys. Commun. 59, 339 (1990)].

[10] A. Delin and E. Tosatti, preprint arxiv.org/abs/cond-mat/0305658 
[11] N. Zabala, M. J. Puska, and R. M. Nieminen, Phys. Rev. Lett. 80, 3336 (1998); Comment and Reply, ibid. 823000 (1999).

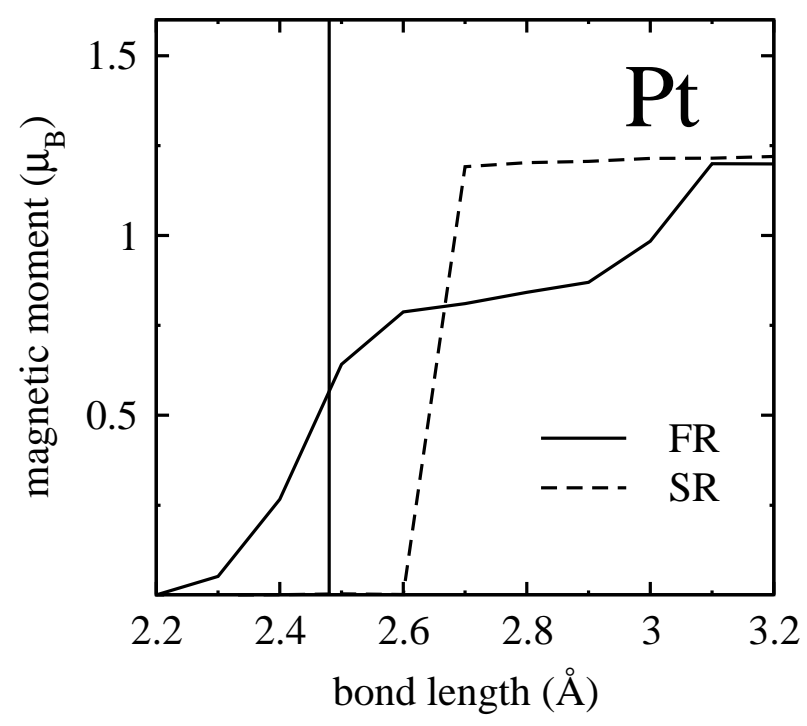

Figure 1: Magnetic spin moment per atom as a function of bond length for a long, monatomic wire of Pt. The vertical line points out the equilibrium bond length. $\quad(\mathrm{FR}=$ fully relativistic calculation; $\mathrm{SR}=$ scalar relativistic calculation). See also Ref. [10]. 

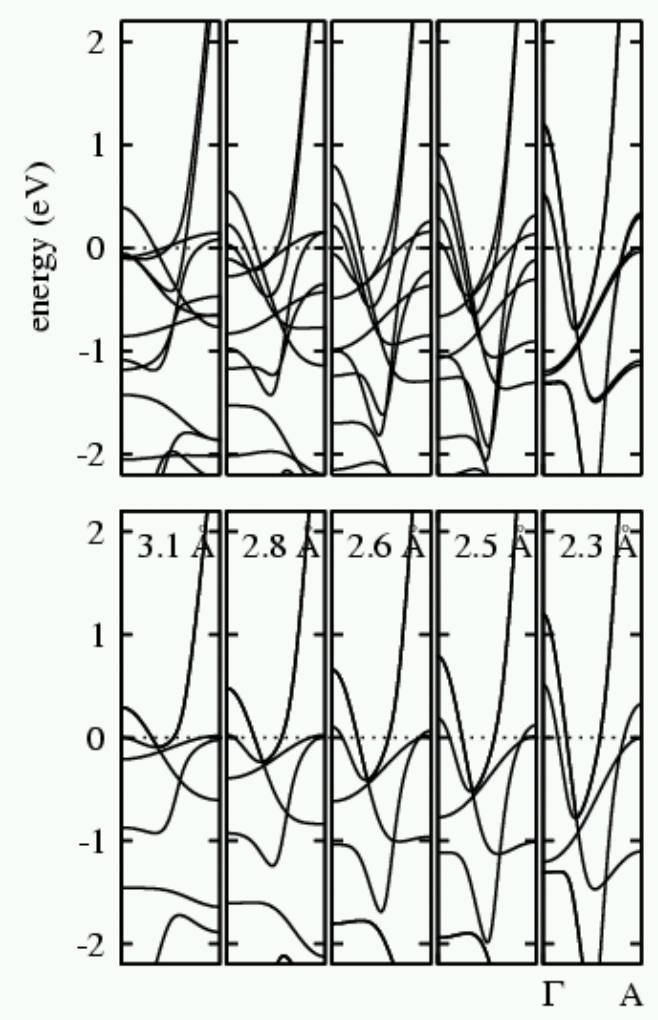

Figure 2: Fully relativistic Pt wire band structures, along the wire direction, for the five bond lengths $3.1 \AA, 2.8 \AA, 2.6 \AA, 2.5 \AA$ and $2.3 \AA$. The Fermi energy is at zero. The upper panels show the ferromagnetic case, and the lower panels the nonmagnetic case. 

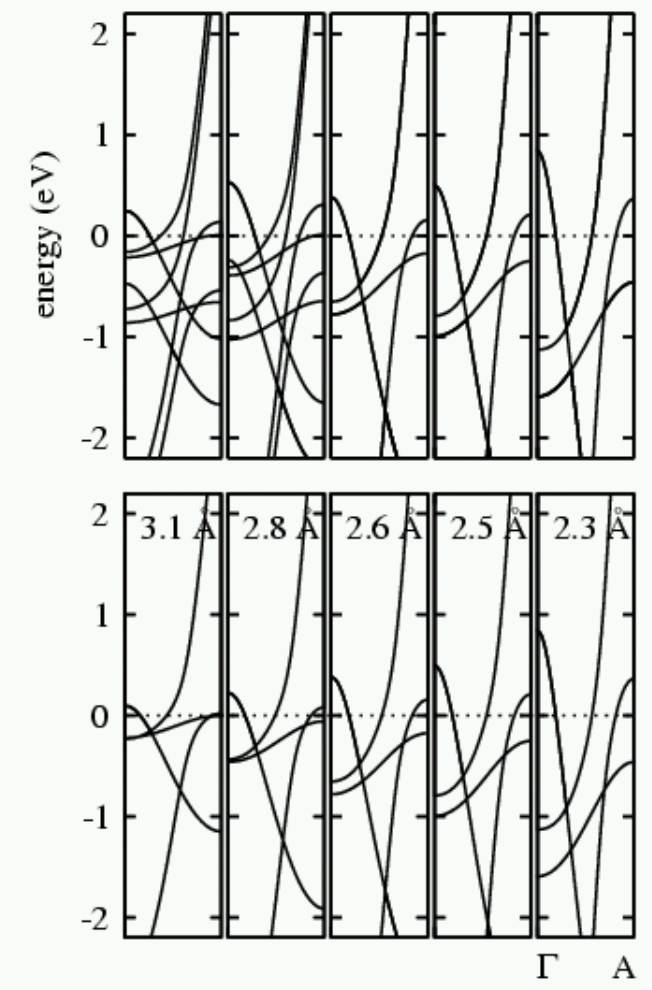

Figure 3: Scalar-relativistic band structures, along the wire direction, for the five bond lengths $3.1 \AA, 2.8 \AA, 2.6 \AA, 2.5 \AA$ and $2.3 \AA$. The Fermi energy is at zero. The upper panels show the ferromagnetic case, and the lower panels the nonmagnetic case. 


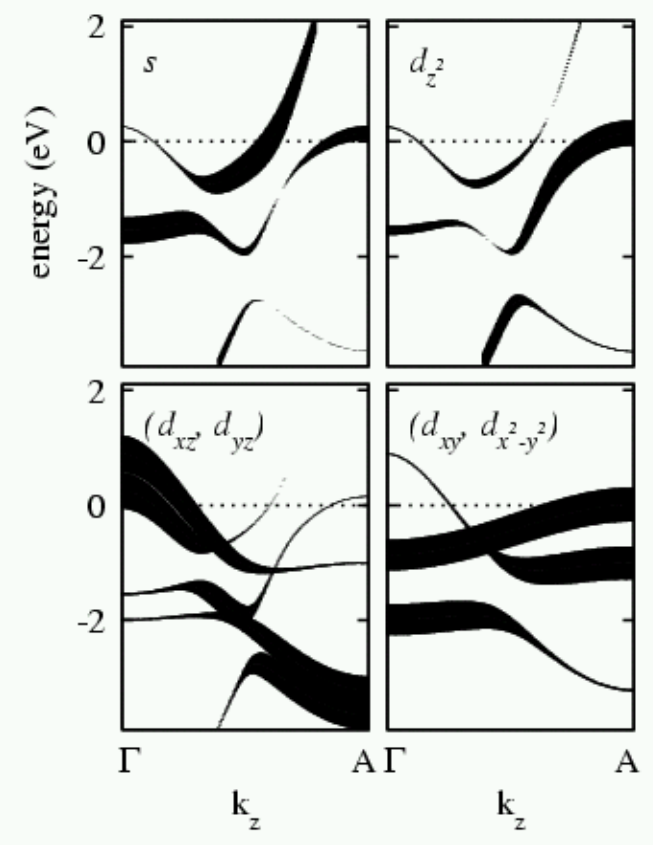

Figure 4: Character-resolved fully relativistic band structure along the wire direction, for nonspinpolarized Pt with a bond length of $2.5 \AA$. The Fermi energy is at zero. See also Ref. [10]. 